\title{
Stellar enhancement factors in a parity dependent approach
}

\author{
L. Huther*1, H.P. Loens ${ }^{1}$, G. Martinez-Pinedo ${ }^{1}$ and K. Langanke ${ }^{1,2,3}$ \\ E-mail: 1.hutheregsi.de \\ ${ }^{1}$ GSI Helmholtzzentrum für Schwerionenforschung, Planckstr. 1, \\ Darmstadt, Germany \\ ${ }^{2}$ Technische Universität Darmstadt, Schlossgartenstr. 7, \\ Darmstadt, Germany \\ ${ }^{3}$ Frankfurt Institute for Advanced Studies, Ruth-Moufang-Str. 1, \\ Frankfurt, Germany
}

\begin{abstract}
Due to the finite temperature of the astrophysical environment stellar neutron capture may proceed from excited states. To account for this effect reaction rates measured in laboratories worldwide are corrected by the stellar enhancement factors. We have calculated these stellar enhancement factors in a parity dependent statistical model approach. The effect of these calculations is rather small, but not negligible. Thus to gain an overall better description, especially for the determination of the solar r-process abundance pattern, the parity dependence is an important aspect for s-process calculations.
\end{abstract}

11th Symposium on Nuclei in the Cosmos, NIC XI

July 19-23, 2010

Heidelberg, Germany

\footnotetext{
* Speaker.
} 


\section{Introduction}

Today the abundance pattern of elements heavier than 56 in our galaxy is believed to be produced by two main processes namely the s- and the r-process, which contribute each about half to the total abundance. While the latter process is related to high neutron densities and temperatures, the s-process is believed to occur at rather low neutron densities either in helium core burning and carbon shell burning in massive stars $\left(M>8 M_{\odot}\right)$ or in hydrogen shell burning and in thermal pulses in AGB stars $(1-3 M \odot)$. The temparatures related to these sites range from 8 to $90 \mathrm{keV}$.

The s-process runs through a sequence of beta decays and neutron capture reactions. Due to the low neutron densities beta decays occur faster than neutron captures. Therefore the path of the s-process is rather close to the valley of stability. This has the advantage that most neutron capture rates could be measured directly in facilities as $n \_$tof or evaluated from folding the corresponding cross section with a maxwell boltzman distribution.

However, in a stellar environment the neutron capture rate can occur differently than in the laboratory, due to the finite temperature of the system that can excite the target nucleus. To describe these modifications arising from the thermal excitation of the target nucleus the so called stellar enhancement factors (SEF) are introduced. The SEF are therefore defined as

$$
\operatorname{SEF}(T)=\frac{\langle\sigma v\rangle_{\text {stel }}}{\langle\sigma v\rangle_{\text {lab }}}
$$

Where $\langle\sigma v\rangle_{\text {stel }}$ is the neutron capture rate in a stellar environment and $\langle\sigma v\rangle_{\text {lab }}$ the corresponding capture rate without considering thermal excitations in the target nucleus.

To obtain these SEF we used the statistical model in the Hauser-Feshbach picture. In this description the cross section is calculated by the formation of a compound nucleus. A neutron capture reaction

$$
i^{\mu}(n, \gamma) m^{v}
$$

where $i$ is the target nucleus in the $\mu$-th excited state, is described in two steps. First, the target reacts with a neutron to form a compound nucleus at an excitation energy $E$ with spin $J$ and parity $\pi$. This compound nucleus then decays in a second step to a daughter nucleus $m$ in its $v$-th excited state and an ejectile $o$. The cross-section for a reaction as given in equation 1.2 can then be described by:

$$
\sigma_{i, j}^{\mu, v}(E)=\frac{\pi \hbar^{2}}{4 \mu_{i, j} E} \frac{1}{\left(2 J_{i}^{\mu}+1\right)} \sum_{J, \pi}(2 J+1) \frac{T_{j}^{\mu}(E, J, \pi) T_{o}^{v}(E, J, \pi)}{\sum_{c} T_{c}(E, J, \pi)} .
$$

The nominator of equation (1.3) is a summation over all possible decay channels of the compound nucleus [1]. The transmission coefficients are calculated by means of an appropriate optical potential. Furthermore experimental unknown states are described via a level-density such as the back-shifted Fermi gas [3].

\subsection{Parity dependence}

Traditionally statistical model calculations assume a level-density description where both parities have equal probabilities $[2,3]$. In general this assumption is not valid at low energies due to nuclear structure effects, i.e. pairing effects. 
To obtain a parity dependent description of the statistical model one has to consider two distinct parts in the statistical model description.

Parity dependent level density As long as states are experimentally known, parity and spins of the excited states can be given as a dirct input for the calculation such as the RIPL database [6]. However if we switch to a level-density description, it has to be both parity and spin dependent. While for the latter dependence statistical descriptions exist and have in general a good agreement with experimental data, it is rather complicated to find a statistical description for the parity behavior of the level-density due to the reasons mentioned above. Therefore Hilaire et al. calculated the level-density by means of a combinatorial model based on HFB single pariticle energies [4]. These level-densities have the huge advantage that due to this microscopic description the obtained level-density is really spin- and paritydependent.

In this work we used 2 different models for the level-density. The first is the above mentioned level density by Hilaire et al. while the second model is the back-shifted Fermi gas (BSFG) model as described with the parameters given in [3]. Contrary to the combinatorial level-density the BSFG model is a macroscopic model that is not able to resolve to a specific parity. Therefore we created a parity projection factor from the combinatorial level-density by

$$
\Pi(E, \pi)=\sum_{J} \frac{\rho_{\text {comb }}(E, J, \pi)}{\rho_{\text {comb }}(E, J, “-“)+\rho_{\text {comb }}\left(E, J, “+{ }^{*}\right)},
$$

where $\rho_{\text {comb }}$ the combinatorial level-density describes.

Optical potential By using parity dependent level densities a huge step towards a parity dependent statistical model is done. However in the calculation of the transmission coefficients of equation 1.3 the parity would be neglegted because global optical potentials are not capable of taking parity into account. Until today no global parity dependent optical potential exist. To overcome this drawback, the authors of [5] modified the transmission coefficient as

$$
T(E, J, \pi)=\beta(E, \pi) \hat{T}(E, J)
$$

where $\hat{T}$ describes the transmission coefficient obtained with a global (parity independent) optical model. Due to the linear dependence of the average transmisiion coefficient with the level-density the factor $\beta$ can be given as

$$
\beta(E, \pi)=2 * \frac{\rho_{\text {comb }}(E, \pi)}{\rho_{\text {comb }}(E, " “-“)+\rho_{\text {comb }}(E, “-“)} .
$$

The factor 2 accounts for the proper normalisation and $\rho_{\text {comb }}(E, \pi)=\sum_{J} \rho_{c o m b}(E, J, \pi)$.

When considering both parts, one obtaines a parity dependent description in the statistical model.

\section{Results}

We calculated the SEF for all s-process nuclei ranging from magnesium up to bismuth for both level-density descriptions above. The results for a temperature of $\mathrm{T}=30 \mathrm{keV}$ can be seen in 


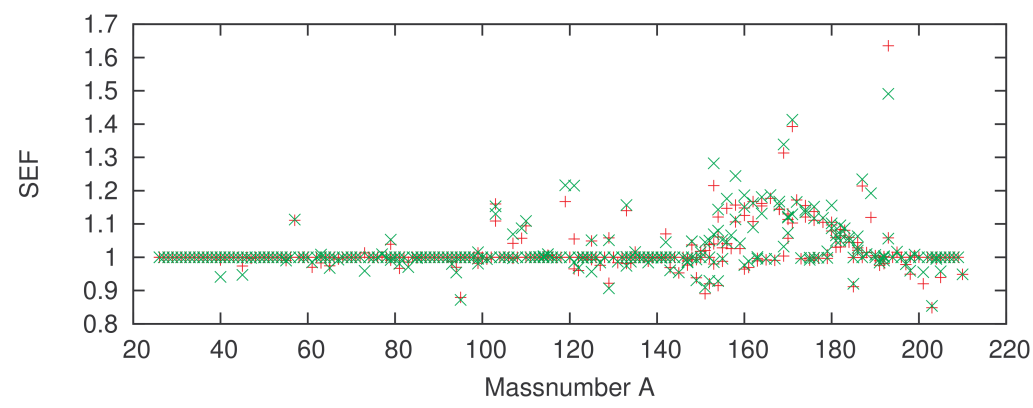

Figure 1: The SEF at an temperature of $30 \mathrm{keV}$ for both level-density descriptions used in this work. Note that both calculations were obtained in the full parity dependent approach.

figure 1 where it can be observed that differences in the SEF arise due to the different level density descriptions.

To show the differences due to the new included parity dependence we present the ratio of SEF obtained in the full parity dependent approach with the SEF in the normal parity independent model. The results can be seen in figure 2 also for a temperature of $30 \mathrm{keV}$, up to $2.5 \%$. These changes do not seem very large, still they are in a few cases not negligible and may increase the exactness of s-process network calculations for r-process abundance patterns. However one can see that these changes are rather small in comparison to the changes due to the different level-density descriptions.

A rather interesting case is the neutron capture reaction on ${ }^{95} \mathrm{Zr}$. As it can be seen in figure 2 this is the reaction for wich we find the largest effect due to the improved parity treatment. The change can reach up to $5 \%$ for temperatures higher than $30 \mathrm{keV}$. However this is only due to the first excited state at a small excitation energy in ${ }^{95} \mathrm{Zr}$, whose existence is questionable [7]. If we remove this state in our calculations the SEF do not change from unity.

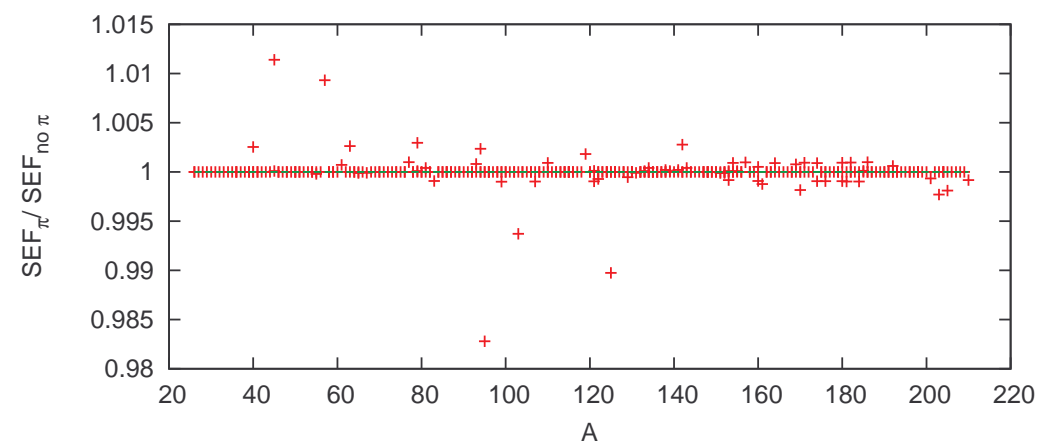

Figure 2: The ratio of the SEF obained in the parity dependent model with the parity independent statistical model. 


\section{Outlook}

Although the changes due to the new included parity dependence are very small, they are not negligible. It is obvious that parity has to be conserved in statistical model calculations. Here we have presented a way of including parity while still using global optical potentials. A special attendance has been laid on the case of ${ }^{95} \mathrm{Zr}$ where the first excited state gives an change up to $5 \%$ in the SEF. This case states explicitly, that for the calculation the excited states of the target nucleus have to be known very well. The largest source for errors in SEF calculations are still the different level density descriptions as can be seen in figure 1, therefore a microscopic level-density description is welcome.

\section{References}

[1] W.Hauser and H.Feshbach, Phys. Rev. 87, 366 (1952), 1952PhRv...87..366H, The Inelastic Scattering of Neutrons

[2] F.-K.Thielemann, J. W.Truran and M.Arnould , Advances in Nuclear Astrophysics, 525 (1986), 1986ana..work..525T, Thermonuclear reaction rates from statistical model calculations

[3] T.Rauscher, F.-K.Thielemann and K.-L.Kratz, Phys. Rev. C 56, 1613 (1997), 1997PhRvC..56.1613R, Nuclear level density and the determination of thermonuclear rates for astrophysics

[4] S.Hilaire and S.Goriely, Nuclear Physics A 779, 63 (2006),2006NuPhA.779...63H,Global microscopic nuclear level densities within the HFB plus combinatorial method for practical applications

[5] H. P.Loens, K.Langanke, G.Martínez-Pinedo, T.Rauscher, and F.-K.Thielemann, Physics Letters B 666, 395 (2008), Complete inclusion of parity-dependent level densities in the statistical description of astrophysical reaction rates, 2008PhLB..666..395L

[6] R.Capote, M. Herman, et al., Nuclear Data Sheets 110, 3107 (2009), RIPL - Reference Input Parameter Library for Calculation of Nuclear Reactions and Nuclear Data Evaluations, 2009NDS...110.3107C

[7] K.Sonnabend, P.Mohr, et al., Phys. Rev. C, 68,048802 (2003), First excited state of the $s$-process branching nucleus ${ }^{95} \mathrm{Zr}$,PhysRevC.68.048802 